\title{
UNA APROXIMACIÓN A LA DIVERSIDAD DE PERSPECTIVAS EN TORNO A LA ATENCIÓN DEL SUFRIMIENTO PSÍQUICO DESDE EL ARTE.
}

\author{
AN APPROACH TO THE DIVERSITY OF PER- \\ SPECTIVES REGARDING THE CARE OF PSY- \\ CHIC SUFFERING FROM ART
}

\begin{abstract}
UMA ABORDAGEM PARA A DIVERSIDADE DE PERSPECTIVAS SOBRE A ATENÇÃO DO SOFRIMENTO PSÍQUICO DESDE A ARTE
\end{abstract}

\section{Resumen}

Este trabajo forma parte de una investigación sobre alternativas de atención a la salud mental a través del arte en un hospital neuropsiquiátrico de la República Argentina.

Durante un año se desarrolló un trabajo de campo

Sy, Anahi

Licenciada en Antropología y Doctora en Ciencias Naturales por la Facultad de Ciencias Naturales y Museo de la Universidad Nacional de La Plata.

Instituto de Salud Colectiva. Universidad Nacional de Lanús. CONICET, Buenos Aires, Argentina. e-mail.anahisy@gmail.com mailto:anahisy@gmail.com

Trabajo recibido: 31 de agosto de 2015. Aprobado: 3 de mayo de etnográfico en tres espacios del hospital que ofrecen diversos talleres de arte. Dicha investigación permitió observar la diversidad de concepciones desde las cuales puede usarse el arte para abordar el sufrimiento psíquico en el contexto del hospital. El objetivo en este trabajo fue analizar y describir las concepciones desde las cuales cada uno de tales espacios trabaja con el arte, profundizando en uno de ellos por el carácter diferencial de la propuesta.

Se parte del supuesto que diferentes concepciones de trabajo con el arte tendrán un impacto directo sobre las prácticas, esto es, cómo se desarrollan los talleres, habilitando ciertas posibilidades de trabajo creativo y/o terapéutico y limitando otras. Metodológicamente se trabajó con observaciones y entrevistas; profundizando en el análisis de uno de los espacios, donde se observa que no se trabaja con el diagnóstico psiquiátrico del 
paciente, y la mirada deja de ser clínica para transformar al "enfermo” en sujeto con capacidad de actuar en relación a su propia salud.

Palabras Clave: Salud Mental, Arte, Antropología Cultural, Psiquiátrico.

\section{Abstract}

This work is part of a research on alternatives of mental health care through art in a mental hospital in Argentina.

During a year, an ethnographic field work was developed in three areas of the hospital which offer different art workshops. Such investigation has permitted the observation of the diversity of conceptions from which art can be used to approach psychic suffering in the hospital setting. The objective of this work was to analyze and describe the conceptions from which each of these areas work with art, deepening in one of them due to the differential character of the proposal.

We start from the supposition that different work conceptions with art will have a direct impact on the practices, this is, how workshops are developed, enabling certain creative and/or therapeutic work possibilities and limiting others. The work was conducted through observations and interviews; going deeply into the analysis of one of the spaces, where it is observed that the patient is not approached from the psychiatric diagnosis, and the look stops being clinical and the "sick person" becomes an individual with the ability to act with regard to his/her own health.

Key words: Mental Health, Art, Cultural Anthropology, Psychiatric.

\section{Resumo}

Este trabalho faz parte de uma pesquisa sobre alternativas aos cuidados da saúde mental através da arte em um hospital neuropsiquiátrico da Argentina.

Durante um ano foi levado a cabo um trabalho de campo etnográfico em três áreas do hospital com o desenvolvimento de várias oficinas de arte. Tal investigação permitiu observar a diversidade das concepções desde as quais a arte pode ser utilizada para tratar o sofrimento mental no contexto do hospital. O objetivo deste estudo foi analisar e descrever as abordagens a partir das quais cada um desses espaços trabalha a arte, aprofundando em um deles pela natureza diferencial da proposta.

Baseia-se no pressuposto de que diferentes concepções de trabalho com a arte terá um impacto direto nas práticas, ou seja, como as oficinas são desenvolvidas, gerando certas possibilidades de trabalho criativo e / ou terapêutico e limitando outras. Metodologicamente, pesquisou-se com observações e entrevistas; aprofundando a análise de uma das áreas onde deve-se salientar que não se trabalha com o diagnóstico psiquiátrico do paciente, e o olhar deixa de ser clínico para transformar o "doente” num sujeito com capacidade de agir em relação à sua própria saúde.

Palavras-chave: Saúde Mental, Arte, Antropologia Cultural, Psiquiátrico.

\section{Introducción}

Este trabajo surge de la investigación desarrollada en un Hospital Psiquiátrico de la ciudad de Buenos Aires (Argentina) con una beca posdoctoral de Consejo Nacional de Investigaciones Científicas y Técnicas (CONICET), que indagó sobre las alternativas de cuidado y atención a la salud mental a través del desarrollo de actividades artísticas.

En la actualidad, en el Hospital se observa la coexistencia de diversas modalidades de atención a los pacientes. A los tratamientos con psicofármacos y mediante psicoterapia se suman diversas actividades, impulsadas por profesionales provenientes de diferentes 
disciplinas, que van desde la terapia ocupacional, hasta la comunicación social y las artes, entre otras. Entre estas actividades, algunas buscan favorecer la enseñanza de oficios (como el "Taller protegido") o la organización de diversas actividades artesanales (como la de "Molineros del Borda"), que a la vez que representan un ingreso económico, permiten trabajar con la externación de los pacientes; y otras que promueven diversas expresiones comunicativas (Radio La Colifata) o artísticas (Frente de Artistas del Borda o el Centro Cultural del Borda, entre otras).

En principio, se puede describir estos grupos de trabajo con características y perspectivas diferentes a las biomédicas, si bien entre ellos también presentan diferencias no solo en las prácticas que promueven sino también en las ideas que subyacen al desarrollo de las mismas. Se puede observar como factor común una orientación hacia la "reinserción social” de los usuarios, buscando favorecer su externación. Sin embargo, los modos de entender esa "reinserción social” y la "externación” son diferentes, cuestión que puede observarse no sólo discursivamente, sino también a través de la dinámica que adquiere cada actividad. Si bien todas ellas presentan diversos grados de dependencia y aval institucional, lo cierto es que estas alternativas co-existen al interior de la misma institución, lo que implica la presencia de una diversidad de significados y prácticas que a simple vista pueden resultar similares, si bien se diferencian e incluso algunas se presentan en abierta oposición con el marco institucional en que se desarrollan.

En este contexto, gran parte de los pacientes internados, y algunos que han sido externados, organizan su cotidianeidad y rutinas, mostrando diversos grados de involucramiento con las mismas, seleccionando unas y descartando otras.

El objetivo en este trabajo es analizar y describir las concepciones desde las cuales se trabaja con el arte en tres espacios diferentes que funcionan en un hospital psiquiátrico, profundizando en uno de ellos por el carácter diferencial de la propuesta. Se parte del supuesto que diferentes concepciones de trabajo con el arte tendrán un impacto directo sobre las prácticas, esto es, cómo se desarrollan los talleres, habilitando ciertas posibilidades de trabajo creativo y/o terapéutico y limitando otras.

Este trabajo se basa en una investigación etnográfica que abarca tres ámbitos del hospital donde se desarrollan actividades artísticas, en los cuales se implementó las técnicas de observación y entrevistas, analizando diferencias y similitudes en las perspectivas y practicas desde las cuales se trabaja con el arte. La elección de dichos espacios estuvo dada, en principio, por nuestro acceso a los mismos, luego, al visualizar las diferentes perspectivas y la forma en que se organiza el trabajo, aun cuando cada uno de ellos propone el trabajo con el arte en el espacio del hospital. Entre las propuestas identificadas se profundizó en el análisis de una de ellas, un colectivo de artistas y profesionales que llamaremos Frente de Artistas (FA), ya que el mismo aparece como expresión de una marcada oposición y crítica hacia el modelo de hospitalización vigente.

Sus integrantes -artistas, psicólogos y psicólogos sociales- no se identifican con las propuestas tradicionales del "arte terapia” o terapia por el arte, esto es, con el uso del arte como un medio para lograr fines específicos de alcance individual: dilucidar diagnósticos, mejora de la psicomotricidad, expresión y elaboración de conflictos psíquicos o comunicación no verbal, entre otros. En este espacio el arte se convierte en instrumento orientado a la transformación institucional de las prácticas de atención en salud mental $\mathrm{y}$, en consecuencia, de las estructuras ideológicas que contribuyen su mantenimiento, al tiempo que a promover la creación de lazos sociales para los usuarios dentro y fuera de las instituciones, favoreciendo la producción de transformaciones individuales, colectivas y sociales (1). En este sentido, no es que se elimine el aspecto terapéutico, sino que se considera que, en cualquier caso, éste ocurre a consecuencia del proceso de trabajo antes descripto. 
Es en relación con esto que se plantea la hipótesis que aquellos espacios que parten de colocar en el lugar de enfermo/paciente/objeto de cuidados a quienes han recibido un diagnóstico psiquiátrico, promueve cierta manera de elaborar la propia subjetividad: como enfermos sujetos pasivos de cuidado. Del mismo modo que otorgar un espacio diferente a quienes sufren un padecimiento psíquico, dónde el diagnóstico no tiene lugar en relación a la actividad que se propone, donde es la propia iniciativa la que adquiere protagonismo, conduce a una elaboración subjetiva que supone posibilidad de actuar sobre la propia realidad, desafiando vulnerabilidades y afirmando espacios propios.

Con esto, no es el interés plantear una discusión en torno a la arbitrariedad del diagnóstico, desacreditar otros espacios terapéuticos diferentes a los del FA. Lo que se busca es conducir a una reflexión más amplia en torno a los lugares desde los que se plantea la atención y cuidado de la salud cuando se trabaja con el arte; a la vez que avanzar en relación al establecimiento de espacios de afirmación de la salud, por contraste con aquellos que parten de la atención a la enfermedad, identificando aspectos que trascienden al nivel individual, para pasar al colectivo /grupal que repercute de forma directa sobre el primero.

\section{Aspectos Metodológicos}

El estudio es de tipo cualitativo, desarrollado a partir de una metodología y técnicas propias del trabajo etnográfico en el ámbito de un hospital público de atención neuropsiquiátrica (cuya identificación se prefiere reservar). Para el análisis se toman tres espacios diferentes, considerando que cada uno de ellos puede ser tomado como caso-ejemplo de una perspectiva particular de trabajo con el arte. Se desarrolló un trabajo de campo de un año (desde mayo de 2010 hasta mayo de 2011) aplicando las técnicas de observación (llevando un registro escrito de las mismas en un cuaderno de campo) y entrevistas (registrando el audio digitalmente, con consentimiento previo del entrevistado/a).

A continuación se describe cada uno de los espacios seleccionados y las técnicas aplicadas:

Espacio 1: se trata del FA., cuya dirección está a cargo de un artista. Se realizó inicialmente una entrevista al mismo, con una duración aproximada de $100 \mathrm{~min}$. En este marco se acordó la participación de la asamblea del grupo para presentar el proyecto a los integrantes y solicitar su autorización para el trabajo. Una vez aceptada, se ccomenzó realizando observación participante en los talleres de "Desmanicomialización”, "Teatro participativo”, "Plástica”, “Música”, “Expresión corporal y danza”, “Letras” y "Fotografía”, durante un periodo de tres meses. Luego se asistió a los talleres de "Circo" durante tres meses y "Teatro" durante diez meses (cada taller tiene una frecuencia semanal con una duración aproximada de 150 min cada día, si bien hubo algunos días en que la permanencia se extendía). Asimismo, acompañamos en el desarrollo de diversas presentaciones fuera del hospital (Jornadas Simultaneas de Arte y Salud Mental, los días 6 y 7 de Agosto de 2010, el Encuentro de arte, derechos humanos y desmanicomializacion, organizado en el marco del Congreso Internacional de Salud Mental y Derechos Humanos el 20 de noviembre de 2010 y en la presentación de la obra de teatro "Reinsertón" durante julio de 2011 en el Centro Cultural IMPA, entre otras actividades fuera del hospital). Se realizaron además entrevistas a 7 (siete) de los coordinadores de talleres del FAB. Cada una de las entrevistas realizadas tuvo entre 90 y 120 min de duración.

Espacio 2: se trata de un ámbito destinado especialmente al desarrollo de actividades artísticas dentro del hospital, su dirección se encuentra a cargo de un médico psiquiatra. En 
ese espacio, entre agosto y diciembre de 2010 se realizaron 5 (cinco) observaciones de 2 a 3 hs. cada una, en esas instancias además se contactó a una referente y, a través de ella, al director del Centro, para acordar una entrevista. La entrevista con el director se concreta en diciembre de 2010, con una duración aproximada de 90 min. Asimismo, se ha tomado información sobre el centro del blog oficial, donde hay publicados materiales escritos y audiovisuales con entrevistas a integrantes del equipo y al director.

Espacio 3: se trata de un ámbito donde se desarrollan diferentes talleres de arte, que funcionan dentro de un servicio del hospital, organizados por el jefe de servicio, de profesión psicólogo. Las instancias de observación en este espacio fueron muy reducidas, no pudo accederse a la observación directa de los talleres, solo se realizaron observaciones en aquellas instancias que buscábamos contactar algún paciente o esperando al jefe del servicio, concertando en ese marco la entrevista, que tuvo una duración aproximada de $120 \mathrm{~min}$.

Se realizaron además entrevistas a 9 (nueve) usuarios, algunos entrevistados en más de una ocasión - solicitando previamente a cada uno de ellos la firma de un consentimiento informado, donde se explicaba las características del proyecto, la posibilidad de elegir participar voluntariamente en este estudio y la libertad plena de no aceptar participar (sin que ello lo afecte de alguna forma); como así también sobre la posibilidad de retirarse del estudio en cualquier momento. Se aclaró además que la información que diera es confidencial y anónima-. Cada una de las entrevistas realizadas tuvo una duración aproximada de entre 90 y 120 minutos, solicitando al entrevistado que eligiera el espacio donde quisiera realizar la entrevista y su autorización para el registro del audio.

Si bien los resultados presentados en este trabajo focalizan en el análisis de las entrevistas realizadas a los referentes de cada uno de los espacios analizados, para realizar una interpretación contextualizada del registro discursivo se toman elementos provenientes de las observaciones. Respecto de la técnica de observación, ésta permite acceder a las actividades cotidianas que se desarrollan, en su contexto "natural" -más allá del significado cultural y/o social que cada actor le atribuye- (2), en nuestro estudio permitió además visualizar la riqueza semántica que emerge en el contexto de cada encuentro, más allá de la palabra que circule y/o de los productos que se proponga elaborar, especialmente vinculada a disposiciones y dinámicas propias de cada grupo. La técnica de observación es clave en el relevamiento de datos etnográficos e implica el involucramiento del observador (investigador) en las actividades cotidianas del grupo, lo que favorece una aproximación a las prácticas y expresiones no verbales tanto físicas como de sentimientos, permitiendo comprender las modalidades que adquieren las interacciones y la comunicación entre los participantes, así como contrastar aquello que proviene de las entrevistas, acerca de lo que se dice que se hace -posibilitando el acceso a lo que efectivamente se hace- (2 y 3).

Todas las observaciones y entrevistas fueron transcriptas de manera textual en archivo Word office y se utilizó como soporte para el análisis el software QRS NVivo 8, programa diseñado especialmente para el análisis inductivo de datos cualitativos, que permite almacenar documentos (material empírico) para su codificación en categorías de análisis, facilitando la exploración de los datos y la elaboración de nuevas preguntas e hipótesis. Se desarrolló un análisis inductivo de los datos provenientes de las entrevistas y observaciones, partiendo de ciertas categorías analíticas que conjugaban nuestro interés teórico y una sistematización preliminar de los datos observacionales, las mismas luego se modificaron, subdividieron y redefinieron. En este trabajo se presentan los resultados vinculados a los siguientes ejes de análisis (aplicados al discurso de los referentes de cada uno de los espacios analizados): 
- $\quad$ Concepciones y usos del arte en el campo de la salud mental y en el ámbito del Hospital en particular

- $\quad$ Formas en que aparecen representados los usuarios: "pacientes"/ "sujetos”/ "enfermos"/ otras.

- $\quad$ Representaciones sobre el cuidado de la salud mental y objetivos de la propuesta que desarrollan.

Para las entrevistas realizadas a los usuarios se tomó el eje: Concepciones de salud/salud mental

En cuanto a los recaudos éticos del estudio, previo a la formalización de la solicitud de autorización firmada a las autoridades del Hospital, el proyecto fue presentado en el espacio que el FA prevé para ello: la Asamblea, en la que participan talleristas y los coordinadores de talleres, así como el director del FA, ahí se consensua la aceptación o no de cualquier proyecto, luego de su presentación. En nuestro caso se describió en qué consistiría nuestra investigación y su finalidad, los participantes realizaron preguntas y, en base a ello, se decidió su aceptación. Luego de esta instancia, se elevó por nota la solicitud a las autoridades del hospital, obteniendo la autorización firmada de la dirección para desarrollar el proyecto.

\section{Aspectos conceptuales: arte y salud mental}

El uso de terapias expresivas en salud mental inicialmente ocurre dentro de una orientación psicoanalítica, en este contexto la expresión por medio del arte, especialmente la pintura, se visualizaba como herramienta diagnóstica o para conocer más acerca de la enfermedad mental. Uno de los primeros que refiere al uso del arte en el contexto de la clínica es Max Simón (1876) sugiriendo la utilidad de las producciones artísticas de los enfermos para diagnosticar sus trastornos mentales o cerebrales. En esa época también se difunde la obra de Lombroso, quien interpreta las creaciones de los pacientes como “representaciones atávicas”, primitivas, regresivas (4 p.32). Hacia 1920, el Psiquiatra e historiador de Arte H. Prinzhorn reúne una colección de casi 5000 pinturas, dibujos, manuscritos, objetos y collages hechos por pacientes de hospitales psiquiátricos en toda Europa, creados entre 1890 y 1920 (Colección de Heidelberg), señalando que estas obras habrían surgido, de la necesidad imperiosa de los pacientes por poner orden al caos, y una pulsión hacia la expresión (4) y (5).

Posteriormente, la orientación más psicoanalítica confluye con terapias de orientación humanista, gestáltica o transaccional; especialmente con aquellas vinculadas a las primeras tentativas de humanización de la atención en las instituciones de salud mental. En este sentido, inicialmente, las experiencias internacionales que tuvieron mayor influencia en América Latina fueron las de la psiquiatría del Sector de Francia y especialmente la Psiquiatría Comunitaria Italiana, inspirada por el Doctor Franco Basaglia. La experiencia dirigida por Basaglia (6) realizada en Trieste, logra el cierre del manicomio y la introducción de toda una estructura comunitaria destinada a la atención de la salud mental. La principal contribución de estos movimientos fue el cuestionamiento del modelo psiquiátrico ortodoxo en vigencia hasta entonces, que llevó a América Latina a los ideales de la desinstitucionalización y la construcción de un nuevo modelo de atención basado en la libertad y la autonomía de los usuarios y en la posibilidad de construir una asistencia interdisciplinaria en comunidad.

Tales influencias hicieron que el uso de actividades artísticas como recurso terapéutico en los servicios de salud mental, generalmente, estuviera vinculado a movimientos de reforma psiquiátrica y experiencias de anti-manicomiales. 
En la actualidad, las modalidades más frecuentes de trabajo con el arte se realizan desde el arte terapia, identificando el trabajo artístico como un facilitador de los procesos comunicativos en pacientes con sufrimiento mental, potenciando la expresión corporal (8), favoreciendo las posibilidades de expresión facial y oral, además del desempeño del lenguaje en cuanto a su forma, uso y contenido (9). También hay trabajos que ponen en primer plano la actividad creativa como medio para recuperar el contacto con las emociones muchas veces reprimidas, facilitando de este modo su exteriorización (10). Asimismo, el arte ha mostrado ser un medio para aliviar el sufrimiento psíquico en población migrante (11) favoreciendo su integración social (12); así como para afrontar el estrés (13), depresión y ansiedad en población carcelaria, vinculándolo a una disminución de la reincidencia (14).

En Argentina, las experiencias vinculadas con la producción artística en el contexto de los hospitales psiquiátricos o centros de atención ocupan un lugar marginal en el contexto de las políticas institucionales y se trata de un aspecto poco explorado de modo sistemático. Sin embargo, se puede citar el trabajo desarrollado desde el Servicio de Salud Mental del Hospital Eva Perón (de San Martín), que funda el "Hospital de Día Rodolfo Iuorno”, organizando diversos talleres de actividades artísticas (teatro, música y plástica, entre otras) como modo de promover la expresión subjetiva de los pacientes, a la vez que potenciar el tratamiento psicoterapéutico que reciben ${ }^{1}$. Actividades de este tipo funcionan en otras instituciones aunque, muchas veces, de modo aislado en algún servicio o centro de salud, dependiendo enteramente de la iniciativa y sostenimiento de los profesionales que allí trabajan, en gran parte de forma voluntaria. Otras han encontrado la forma de "institucionalizarse" conformándose como Asociaciones Civiles, como es el caso del Proyecto Suma, que incorpora talleres de arte a una propuesta de abordaje integral de la salud mental en su contexto social y comunitario (15) o el de la Red Nacional de Arte y Salud Mental, que nuclea diversos grupos que trabajan en hospitales de todo el país -entre ellos el Frente de Artistas aquí estudiado- con una orientación “desmanicomializadora”2 y Asociaciones Civiles como la Radio La Colifata y Cooperanza.

Sin embargo, se debe señalar que las diferentes formas en que se promueve el uso de actividades artísticas en el campo de la salud, y el hospital no es la excepción, no siempre se caracterizan por los mismos principios clínicos, estéticos y valores ético-políticos. En la actualidad, en una misma institución psiquiátrica o en distintos centros de atención, los usos del arte son diversos; se puede señalar al menos cuatro orientaciones principales:

- Actividades artísticas ofrecidas como «entretenimiento» u ocupación para el paciente, sin que necesariamente esté provista de algún sentido para él.

- Talleres vinculados a lo que se conoce como "arte terapia” o "terapia por el arte” donde la actividad creativa se convierte en el medio usado para tratar al paciente. Se hallarían dentro de este enfoque el uso de actividades artísticas como recurso terapéutico orientado a dilucidar diagnósticos y/o alcanzar objetivos de tratamiento, la mejora de la psicomotricidad, la expresión y elaboración de conflictos psíquicos, la comunicación no verbal, entre otros (4).

\footnotetext{
${ }^{1} \mathrm{Al}$ respecto puede consultarse: Alazraqui, H. Tratamiento en Hospital de día: relación entre producción individual y capacidad de funcionamiento social. Revista del Instituto de Investigaciones de la Facultad de Psicología UBA, CABA. 2010; 15(2):7- 29. Alazraqui, H. El dispositivo de Hospital de día: historia, evolución y conceptualización. En: Los bordes en la clínica. JVE ediciones. CABA; 1999 (p. 101-110). Alazraqui, H. y Naszewski, M. Algunas puntualizaciones sobre el dispositivo de Hospital de día. Revista Psicoanálisis y Hospital CABA. 1997; 11: 26-29.

${ }^{2}$ El término "desmanicomialización" es un neologismo creado desde el Departamento de Salud Mental del Ministerio de Salud Pública de la Provincia de Río Negro en el año 1988, para nombrar al proceso de transformación en la atención de la salud mental que se estaba implementando en dicha provincia (Cohen, 2007). Este concepto es utilizado en Argentina por gran parte de los grupos que cuestionan el modelo de internación y tratamientos vigentes en los hospitales neuropsiquiátricos, promoviendo un cambio en el proceso de atención a la salud mental, orientado hacia la humanización de la atención psiquiátrica, en base a principios democráticos, pluralistas (en cuanto a los encuadres teóricos y disciplinarios), con un compromiso político y de transformación social.
} 
3 - El uso de actividades artísticas como posibilidad de creación de lazos sociales para los usuarios dentro y fuera de las instituciones, orientado a la producción de transformaciones individuales, colectivas y sociales.

4 - El arte como instrumento de luchas y resistencias políticas con vistas a la transformación de las prácticas de atención en salud mental y a promover un cambio en el status quo social.

La distinción establecida es a los fines puramente didácticos, simplifica la complejidad inherente a cada uno de los enfoques y, en la práctica, esos objetivos se superponen, complementan e incluso pueden ocurrir al mismo tiempo. No se trata de valorar estas diferentes modalidades terapéuticas como mejores o peores, positivas o negativas, solo presentarlas como diferentes posturas profesionales y modos de abordaje clínico que responderán a necesidades diferentes (1).

\section{Resultados}

$\mathrm{Al}$ acceder a tres perspectivas diferentes sobre un mismo tema observamos que la mejor manera de presentarlas es la cita textual que articule perspectivas en torno al mismo eje de análisis (respetando la transcripción literal del registro de audio, cuidando que en la selección de los fragmentos y su articulación no quede cambiado o descontextualizado lo expresado por cada uno de los entrevistados). De esta forma se hacen visibles convergencias, diferencias, contrastes de opiniones y concepciones sobre cada una de las dimensiones de análisis.

Al indagar en torno a las concepciones y el uso del arte en el contexto del hospital se observan diferentes posicionamientos, vinculados además a diferentes formas en que aparecen representados sus usuarios.

\section{Director espacio 2:}

[...] el arte es una herramienta muy importante para el tratamiento de la enfermedad mental [...]. El objetivo es llevar a cabo una propuesta médica, en la que el paciente, ya sea externado o interno, concurra al centro a participar y a desarrollar su expresión creativa, pero a la vez que comparta un momento terapéutico con profesionales y artistas; como valor agregado, el artista participa del momento terapéutico. Las tareas se realizan y supervisan con profesionales, psicólogos y psiquiatras de la institución, a diferencia de otras épocas [...] la meta es que el paciente se rehabilite, encuentre y busque sus posibilidades y se reinserte con algún saber, con una estrategia que surja de él mismo [...]. El arte promueve situaciones de alegría y encuentro de creación en el enfermo mental y es así como algunos pacientes encontraron una cuasi salida laboral a partir de que accedieron al centro cultural. El crecimiento de esta propuesta tiene que ser amplio porque su potencial, también lo es [...] Es fundamental poder darle continuidad al centro, ya que estamos hablando de pacientes enfermos mentales; en la sociedad se habla mucho de recuperación e inserción social pero no se hace nada"

Director espacio 3:

[...] lo que yo propongo es una trama, así como la trama de una tela, donde los distintos dispositivos que son libres, hay otros servicios, lo más importante es que las personas que acompañan este proceso terapéutico o artístico, también tienen otros espacios, no es solo el arte, pero también el arte, está muy vinculado, en el sentido de la invención a la cuestión intelectual, así como el paciente te dice una palabra rara que tiene significado solo para él, es una invención y esa invención le permite a él suplir algo que no encuentra como, algo, lo pulsional, la pulsión es 
un concepto que Freud plantea [...] es a partir de lo psicológico, es un concepto para poder pensar esta vinculación del cuerpo con la realidad psíquica, no. Y es algo de lo que atraviesa el cuerpo, entonces el cuerpo también está absolutamente tomado en la creación artística, no es solo las ideas, a lo largo del empuje pasional, que un poco tiene que ver con el entusiasmo por ejemplo, en el mejor de los casos, tiene que ver con la hostilidad, porque bueno, la posibilidad sublimatoria de poder articular estos y que emerja algo nuevo es propia del artista, el artista se adelanta digamos, para el psicoanálisis también toma la posibilidad del sujeto en el trabajo analítico, entonces hay una concepción de Lacan, que ya no es más la articulación edípica, sino que es mas allá del edipo, el que no ha podido hacer ese atravesamiento edípico encuentra su propia solución, es lo que se llama la articulación, entonces a eso apuntamos en tratamiento con el paciente. [...]

\section{Director espacio 1:}

[...] Por ahí nosotros tenemos algunas diferencias con otras agrupaciones artísticas que lo toman más como arte terapia, el hecho que el paciente haga algo es importante, es importante, pero no es suficiente, (...) yo creo que el Frente de Artistas lo que tiene es la posición política de la destrucción del manicomio, de la no institucionalización del sufrimiento mental, de la no manicomialización, [...] el proyecto de desmanicomialización que es el cierre del manicomio y la atención del sufrimiento mental en Hospitales Generales con tratamientos cortos, internaciones cortas, que atiendan a la vivienda, al trabajo, a los afectos. Nosotros partimos de la idea que el arte tiene elementos conceptuales y prácticos que puede trabajar sobre la subjetividad de las personas y puede producir efectos sobre las personas y en la sociedad, en general el manicomio lo que hace es como, palabra de Pichon Riviere, va haciendo que las personas dejen de ser personas, o sea pierdan la capacidad de hacer, sentir y hacer o crear, el manicomio lo que hace es que las personas que viven y trabajan acá adentro, sobre todo las que viven se van obturando, neutralizando en la pasión, el deseo, la voluntad, la sociabilidad, entonces, dejan de ser sujetos y se convierten en objetos, el arte invita a un trabajo grupal, a un proceso creador, a nuevos vínculos, convoca a poder pensar y sentir nuevas cosas, a poder proyectar, superar los muros, físicos e ideológicos del manicomio, y esa salida es lo que produce un efecto desmanicomializador, porque el momento que se sale no solo sale la producción sino que sale esa voz acallada del Hospital. Esa persona que puede decir a través de su propia producción, podemos decir lo negativo de la institución, o sea denunciar lo negativo de la institución y ahí produce un efecto desmanicomializador en cuanto a la institución, al manicomio, o sea eso que se dé afuera, se replica adentro del manicomio y genera un nuevo movimiento institucional que de otra manera no hubiese podido suceder y aparecen las contradicciones de la institución, el tercer movimiento que produce es lo social, en la medida que la locura circule a través del arte, con una información distinta a la que circula normalmente por la existencia del manicomio, comienza a modificarse ese pensamiento, ese imaginario que tiene la gente y la gente como uno, o sea personas, grupos, instituciones, comunidades, sociedades, comienza a tener una posición más política, más ideológica de la problemática y a tomar una posición mas de participación activa, no religiosa "pobrecito los locos" sino de modificación de cambio de transformación, eso se difiere mucho al arte terapia".

De las citas expuestas, se pueden extraer, inicialmente, diversas formas de pensar el uso del arte en el campo de la salud mental, que podemos visualizar vinculada a la trayectoria profesional del entrevistado. Con ello no se quiere caer en el determinismo de pensar que 
cada vez que un psiquiatra, artista o psicólogo trabaje con el arte lo haga de esta manera, sino que se observa que es la formación recibida (vinculada a cierta trayectoria particular de trabajo con el arte) la que habilita cierta manera de pensar los usos del arte en el campo de la salud mental.

En el relato del psiquiatra (director espacio 2) aparece claramente la "enfermedad mental" como objeto a ser tratado desde una propuesta médica donde el arte es herramienta (medio) y la "expresión creativa" es parte de lo que se considera terapéutico; donde el artista (refiere a quien se encuentre coordinado el taller) "participa del momento terapéutico". Puede observarse la mirada médica del tema, que se traduce además en lo simbólico, donde quedan claramente plasmadas las diferencias entre el artista y el "paciente”, "enfermo mental” que participa de ese espacio de "rehabilitación”. Aquí subyace la idea de sujeto pasivo/objeto de cuidados, que debe volver a ser habilitado por el médico para que su reinserción social efectiva ocurra. Algunos de los aspectos observados en este espacio nos permiten visualizar tales aspectos en lo cotidiano: los profesionales usan guardapolvos, donde colocan además un cartel con su nombre y profesión, el baño del lugar sólo puede ser usado por "los que vienen de afuera" las personas internadas deben ir al servicio del hospital que les corresponda.

En la perspectiva del psicólogo (espacio 3), se visualiza claramente la aproximación desde la psicología, refiriendo a "lo pulsional" y a la posibilidad sublimatoria del trabajo analítico, citando referentes del campo como Lacan y Freud. Desde esta perspectiva, el arte es considerado un medio efectivo para visualizar elementos de la "realidad psíquica", para articular en el "tratamiento con el paciente".

El director del FA (espacio 1), se posiciona claramente por fuera del arte terapia, para manifestar abiertamente su postura a favor del hecho artístico como de la desmanicomialización, esto trasciende su formación de artista, aunque tampoco lo asemeja a cualquiera de las perspectivas antes señaladas. Plantea que su trabajo puede tener un efecto a nivel de la subjetividad de los usuarios, a nivel social y a nivel institucional, que se debería a un proceso grupal creador que genera la posibilidad de salir del hospital con una presentación de lo producido que funciona como critica al encierro manicomial, mostrando las contradicciones del mismo orden institucional y buscando un cambio en el imaginario social de la "locura". Se trata abiertamente de una postura político-ideológica frente al tema, él mismo se presentó en la entrevista como un militante, más allá de su profesión. Es importante señalar que no se refiere a patologías y/o enfermos/enfermedades mentales, como ocurre en los otros casos, sino que refiere a "personas" con "sufrimiento mental".

Respecto a la propuesta y los objetivos de las actividades que se proponen, también se encuentran diferentes perspectivas que se vinculan además a ciertas características que se atribuye o suele atribuir a las personas con sufrimiento mental:

\section{Director espacio 2:}

[...] El (espacio2) cuenta con un blog en el que semanalmente se actualiza la información, pero también tiene una función terapéutica, tratamos de que los pacientes lo vean y se vean ellos sobre todo, para que entiendan que la obra trasciende hacia el afuera, la idea de que el arte y la rehabilitación pueden marchar juntas, además es otra forma de defender a los hospitales psiquiátricos, amenazados por políticas de salud que pretenden acotar su rol, [...] hacer pública esta tarea es defender al hospital por medio del hacer, de hechos concretos y objetivables donde se ve claramente que el paciente participa, disfruta y aprende. La mejor defensa para nuestros hospitales psiquiátricos es que sigan funcionando a través del trabajo con los pacientes [...]. 
Director espacio 3:

[...] mi proyecto se llamó arte, cultura, psicoanálisis y trama social. Y digamos para diferenciar de todas las intervenciones culturales del hospital que lo que hacían era llevar afuera la producción del Hospital, yo arme una cosa distinta, propuse para modificar la estructura hospitalaria introducir a manera de cortes intersticios que produzcan los talleres, artistas, entonces por ejemplo, ya esto lo había hecho antes por eso apoyo mucho y acompaño todos los talleres que tienen que ver con este tipo de trabajo, trajimos el 'Pan del Borda', al teatro municipal, más allá de que los pacientes produzcan cosas [...] no es que los pacientes sean artistas, por ser pacientes o por ser locos, viste, no cualquiera es Artaud [...]

\section{Director espacio 2:}

[...] Algunos de los principales artistas, escritores y pensadores de la historia han padecido de enfermedades mentales. También se sabe que la creatividad está asociada con un mayor riesgo de depresión, esquizofrenia y bipolar [...]

\section{Director espacio 1:}

[...] Hay un romanticismo en el arte [...] nosotros no hacemos ninguna pregunta, ni indagación sobre cuál es el diagnostico o el problema que pueda tener ese paciente, lo que a veces se intenta es que el coordinador psicológico pueda tener una relación con el profesional que atiende a ese paciente por una cuestión de respeto y reciprocidad, porque ese paciente está internado y de alguna manera la responsabilidad recae en los profesionales que lo atienden entonces nuestra relación con los profesionales suele ser operativa, si nosotros vemos que un paciente toca bien la guitarra, pero vemos que por la medicación viene muy contracturado a veces se produce un vínculo con el profesional para ver en qué medida puede ayudar ese profesional para que sea posibilidad rehabilitadora la que produce el FA, lo pueda ayudar además que desde la medicina, en este caso darle menos medicación. O a veces hay profesionales que se interesan, "bueno a mí me interesa que este muchacho pueda salir cuanto antes y que le pueda servir a externarse [...] si por eso no nos interesa mucho el diagnostico mismo, porque creemos que ya está bueno el laburo, por el laburo mismo, está bueno si me hace bien, y está bueno también poner en palabras lo que me hace bien y lo que me hace mal [...] si, algo que creo, que si esto es terapéutico viene después, como que no está antepuesto nosotros intentamos hacer arte y que sea como cualquier espacio artístico, después lo que trae acompañado eso de cada uno trabaje internamente su cuerpo o que salgan algunas cosas a la luz buenísimo, como le pasa a uno que hace un taller de teatro en otro lugar o hace yoga o hace danza, pero el objetivo primero no es la terapia, no es traemos los problemas y en base a eso hacemos algo, cada integrante, uno ya sabe en que ha ido cambiando o como encausarlo, porque somos todos distintos, cada uno tiene sus bloqueos y decir cada uno, bueno, vos podes buscar por acá, vos podes buscar por allá, se tiene mucho en cuenta eso, la individualidad también, así como el grupo, la individualidad, a nivel psíquico y psíquico, de trabajo interno [...]

\section{Director espacio 3:}

[...] después se puede pensar qué es lo que lleva a un sujeto a una internación, yo primero pregunto qué es lo que le ha llevado, que es lo que imposibilita que viva en la sociedad, pero no es solo la sociedad, la institución, la institución tiene puertas abiertas, pero hay algo de la estructura y hay algo de la sociedad, sobre todo el discurso capitalista que lleva a encerrar a quien no respeta las normas sociales, aunque por el otro lado todo vale pero hay quienes no pueden entrar en 
los medios de producción, muchos de los pacientes internados por alcoholismo, psicosis alcohólicas, en el fondo eran unos depresivos que habían perdido su trabajo y habían perdido su familia, ahí no sabías que era primero, si el huevo o la gallina, si era por su propia estructura que no podían sostenerse en el mundo o si las situaciones que los fueron limitando, tenía un puesto importante en el banco y el banco cerro, no, entonces, te digo, la sociedad feroz de capitalismo feroz te lleva a necesariamente a la exclusión [...]

\section{Director espacio 1:}

[...] hay una realidad que estas cosas concretas y materiales si no están calculadas en un proyecto de salud, redes en organizaciones mancomunadas es difícil, hay personas que pueden hacer grandes procesos y sin embargo no pueden salir de acá, por eso en un proceso desmanicomializador seria más complicado y si tuviéramos un programa un poco más serio más consecuente con lo que serían las leyes, seguramente sería mucho más rica la tarea de un lugar como el frente lo que pasa que si hay cosas que no están garantizadas, en relación a la vivienda o al trabajo, es difícil [...]

En las citas se observa que las diferentes propuestas se distinguen en cuanto a los objetivos del proceso de trabajo: mientras que en el FA (espacio 1) es fundamental que quienes participan de los talleres salgan para realizar las presentaciones de lo producido -cuando el trabajo adquiere cierto nivel artístico-, en el espacio 2 "sale y circula” virtualmente la obra, a través de un blog, al que también se le atribuye una función terapéutica, cuando los pacientes tienen la posibilidad de ver que la obra trasciende hacia el afuera. Tal actividad y esa forma de circular es la que el director de dicho espacio atribuye la capacidad de “defender a los hospitales psiquiátricos”, insistiendo sobre la defensa del hospital como espacio de atención a la salud mental por excelencia, sin referencias al marco de la ley de salud mental que da lugar a otras formas de abordaje del sufrimiento psíquico, por fuera del modelo hospitalario vigente.

En el caso del espacio 3, el psicólogo plantea que es la entrada de propuestas externas al hospital lo que podría modificar la estructura hospitalaria, más allá de la producción de "los pacientes"; criticando el lugar que se le da al arte al colocarlo próximo a la locura, en particular a ciertos diagnósticos, en clara diferencia con el Director del espacio 2, quien convierte la creatividad artística en un factor de riesgo de la enfermedad mental, señalando que el artista tiene más posibilidades de padecer este tipo de enfermedades. Tal posicionamiento también es criticado desde el FA, asociado a la idea romántica del loco poseyendo capacidades extraordinarias, desde el FA, se busca eliminar cualquier juicio bio-médico o psicológico a priori respecto del paciente, es en este sentido que podemos considerar que los etiquetamientos y estereotipos se borran para trabajar con la persona, integrada a un grupo, con una propuesta que busca trascender los muros del hospital, una propuesta con un claro posicionamiento ideológico, donde las capacidades de pensamiento crítico, de decir y expresar a través del arte traerán consecuencias a nivel subjetivo, lo que devolverá a la persona sus estatus de sujeto de derechos con capacidad de actuar en relación a su salud (lo que necesariamente ocurre a consecuencia de un cambio en la subjetividad de la persona).

Puede considerarse que son estos sujetos los que podrán hacer uso de las "puertas abiertas” que señala el jefe de servicio (espacio 3). El trabajo desarrollado en el hospital permite observar que, más allá de que se hable de un hospital de puertas abiertas, lo que encierra y cercena las libertades individuales en tales espacios tiene otra naturaleza: química, en los casos de pacientes cuya medicación o sobre medicación les impide ciertas capacidades; económica, porque sería necesaria una reinserción laboral en el contexto social y comunitario del paciente para que la salida del hospital represente una posibilidad 
real y de naturaleza subjetiva, vinculada al tiempo de internación y la forma en que se va modelando y reforzando una identidad asociada al diagnóstico psiquiátrico.

Por último, aparece en las entrevistas una reflexión acerca del origen del problema que aqueja a los usuarios, vinculado a cuestiones de orden social y económico, que busca trascender las explicaciones biologicistas y/o psicologistas, para abordar cuestiones vinculadas a las relaciones y vínculos contradictorios. Dirá el psicólogo que esto "lleva a encerrar a quien no respeta las normas sociales, aunque por el otro lado todo vale, pero hay quienes no pueden entrar en los medios de producción”, planteado el interrogante sobre si se trata de una estructura previa del sujeto, que se expresa en ciertas circunstancias, o si son los contextos y trayectorias personales las que desencadenan este tipo de manifestaciones psicológicas. Desde la perspectiva del FA el énfasis se coloca en las condiciones que favorecen/limitan la salida del hospital, cuando ciertas necesidades básicas, como vivienda y trabajo no se encuentran garantizadas, entrando estas personas en un círculo que las devuelven al espacio manicomial con toda la carga de sufrimiento mental que ello conlleva.

Hasta aquí se puede ver cómo cierta forma de pensar el arte configura una idea de enfermo/enfermedad y las diversas formas en que se considera que el arte puede aportar a un proceso de trabajo en torno al sufrimiento psíquico. A continuación introducimos algunas cuestiones vinculadas a las entrevistas realizadas a los participantes de esos espacios, sobre las que la mayoría de los entrevistados coinciden al pensar la salud, para habilitar la reflexión en torno a la producción de subjetividad:

[...] la salud y la enfermedad para mí son cosas, son parte de la vida, yo creo que la vida es como una cajita, la caja de pandora, o como quieras llamarle, es un casete o un pen drive, es un envase un estuche y dentro hay muchos componentes, muchos ingredientes, donde puede estar la enfermedad, la salud, puede estar el amor, el desamor, pero yo creo que el amor y el desamor tienen mucha incidencia sobre la salud y la enfermedad, creo que a partir del amor podemos vivir muy sanos, y del desamor, tal vez si sabemos barajarlo y manejarlo podemos rescatar bastante [...] si comparto lo que te está pasando para poder ayudarte, todo eso creo que tiene que ver con la salud, contar con otro" (Mario, integrante del FA, internado en el Servicio analizado -espacio 3- y participante de algunas actividades que se organizan en ese espacio)

[...] La cura, salir del encierro y encontrar una vida normal, natural. Una vida cotidiana como puede pasar cualquier persona normal salida del encierro [...] una vida normal es dedicarse a su propio equilibrio. Equilibrio a una sociedad que desconoce, darse cuenta la realidad de la vida" (Juan, integrante del FA, y participante de los talleres del espacio 3) [...]

[...] como la ley 448 de reinserción social y laboral, una forma de pensar un abordaje de la salud diferente a la vinculación del paciente con el médico, la familia con los pacientes, es una forma atravesada por la humanización, donde se respetan los derechos del hombre [...] (Ivan, integrante del FAB y participante del espacio 2)

[...] creo que hay que integrarlo a lo social, vincularlo a lo social [...] (Carlos, participante del espacio 3).

Se Podría señalar que lo que buscan los pacientes es la posibilidad de volver a habitar espacios fuera del hospital, en la sociedad, a través de un trato más humano, amoroso, afectivo, y respetuoso de la legislación vigente. En ese sentido las alternativas deben orientarse hacia la creación de espacios de participación donde se proponga maneras de habitar el mundo y el propio cuerpo, nuevos modos de comunicación y diálogo entre las personas, nuevas formas de expresar deseos, anhelos, luchas y sufrimientos muy profundos, muchas veces imposibles de nombrar o poner en palabras. 
A partir de lo expuesto es posible entrar en diálogo con la literatura producida sobre el tema a fin de discutir los resultados poniéndolos en relación con la hipótesis de trabajo planteada.

Es abundante la bibliografía producida en el campo de la Sociología y Antropología médica que critica la práctica médica por colocar su foco en la enfermedad, aislada del contexto social y cultural en que ésta ocurre, sin tener en cuenta la historicidad de la propia Ciencia Médica. Desde la biomedicina la salud y la enfermedad son conceptos no problemáticos, se definen por los criterios objetivos de la ciencia biomédica y se asume que esta es, efectivamente, la perspectiva autorizada para delimitar las fronteras entre lo normal y lo patológico (16). Este posicionamiento dominó y domina la práctica de la psiquiatría y se expresa aún en propuestas que se pretenden alternativas y/o complementarias de otros tratamientos psiquiátricos. El análisis que se presenta indaga en torno a la forma en que se designa a los usuarios de los espacios de arte analizados y visualizamos que nombrar como "pacientes" o "enfermos mentales" a quienes participan de los talleres, viene asociada a cierta manera particular de concebir -y a la vez modelar- a los usuarios: objetos pasivos de cuidado.

La teoría de la etiquetación (Labeling theory) realiza aportes fundamentales al respecto. Lembert, en su obra Social Pathology (1951) señala que no es la enfermedad en sí, sino la reacción social a la enfermedad la que da cuenta de los principales diferenciales de aquello que llamamos enfermedad. Tal reacción social debe ser validada para ser significativa, y su validación consiste en el control social que se ejerce sobre los individuos desviados, bajo la forma de aislamiento, segregación, castigo y algunas formas de tratamiento organizado. Este trabajo da lugar a diversos estudios, entre los más reconocidos podemos citar el de Becker (1963) Outsiders, quien sostiene que la desviación es construida por los propios grupos sociales al crear reglas sobre quién es el desviado (por tanto quién es enfermo) "es aquel sobre quien tal etiqueta se ha aplicado exitosamente, la conducta desviada es la conducta así etiquetada por los individuos” (18 p.9); en el mismo sentido Scheff (19) sostiene que la mayor parte de las enfermedades mentales constituyen, al menos en parte, un rol social; y que es la reacción social de etiquetación la determinación más importante de entrada al rol de enfermo mental. Waxler (1980) señala que una vez etiquetada como enferma mental una persona puede encontrarse irremediablemente en medio de una profecía auto cumplida, precisamente porque es confinada dentro de un rol específico (16).

En este sentido, Goffman en su libro Internados (17) plantea que la institución psiquiátrica es la que hace la diferencia respecto a lo que llamamos enfermedad mental, señala que no es tanto que esas instituciones traten enfermos mentales, sino a la inversa, llamamos enfermos mentales a las personas que estas instituciones atrapan. Las decisiones de "etiquetado" como patológico o necesitado de una intervención terapéutica y la variabilidad del umbral a partir del cual los individuos pasan a ser considerados pacientes, ha tendido a naturalizarse la en nuestra sociedad.

Sin quitar valor de verdad a lo que la medicina y la psiquiatría en particular tienen para decir y reconocen orgánicamente como etiología de ciertos padecimientos mentales, no se puede negar que el rótulo de "enfermo mental", "paciente" originado desde un conocimiento biomédico, orientado a controlar aquello que se considera indeseable desde los propios patrones socio-históricos y culturales; sitúa al sujeto en un espacio social (el de la enfermedad) y en un rol (el de enfermo). En este sentido, se observa que en los espacios 2 y 3 analizados los sujetos/usuarios de espacios de arte son pensados como enfermos o pacientes objeto de cuidado, reproduciendo viejas estructuras con propuestas "novedosas" o mejor, que diversifican los tratamientos, sin una real transformación de los mismos que se oriente a la superación de las críticas expuestas. Como ha sido ampliamente desarrollado en diversos trabajos sobre la modalidad que adquiere el tratamiento e internación en 
instituciones psiquiátricas, ésta permea el cuerpo de los internos, en este contexto es natural que ocurra una reconfiguración identitaria de la persona: como "interno", marcada por la alteridad y la diferencia. Diferentes experiencias de trabajo muestran que los internos habitan en el hospital como el hospital habita en los internos (20). En este mismo sentido, Correa Urquiza señala:

"En la mayoría de las ocasiones, la carrera en tanto paciente (Goffman, 1989) se transforma a la vez en el eje de un proceso de interiorización de una identidad asociada a la idea de enfermedad. El trastorno y sus calificaciones impregnan el tejido de vida del sujeto que deviene así en una suerte de enfermo absoluto. Ya ni tan sólo está; sino que es enfermo. Y lo es en todas y cada una de las instancias sociales de su yo cotidiano” (22 p. $50)$.

Desarrollos en el campo de la psiquiatría, especialmente en el de la Salud Mental Comunitaria de la última década, proponen un abordaje superador de la mirada que estigmatiza a las personas con sufrimiento mental introduciendo el concepto de "recovery", traducido al español como recuperación. El mismo puede tener varios usos y significados, Slade (22) señala que cuando se refiere a "recuperación clínica”, involucra la remisión de los síntomas así como la reinserción en el espacio social; supone el 'volver a la normalidad' evaluado desde la perspectiva del profesional de la salud mental, mientras que cuando se habla de "recuperación personal" se trata de aquella que emerge de la experiencia de quien ha sufrido alguna enfermedad mental, y está vinculado a un sentimiento profundamente personal respecto a cambios actitudinales, valores, sentimientos, objetivos, habilidades y/o roles. Es un modo de vivir que resulta satisfactorio y esperanzador aun dentro de las limitaciones que causa la enfermedad. De este modo, la recuperación involucra el desarrollo de nuevos significados y propósitos para la propia vida aún dentro de la enfermedad (22). Si bien como se ha señalado previamente, no deja de referirse a la "enfermedad mental" como categoría no problemática y como algo dado sin necesidad de ser sometida a discusión, la perspectiva resulta interesante para pensar la salud psíquica. Whitley y Drake (23) al revisar el concepto de recuperación plantean diferentes dimensiones: a la clínica y existencial se suma una funcional, otra física y una social. Cada una de estas dimensiones incluye lo que generalmente se entiende como recuperación. Agrest y Druetta (15), en un trabajo que analiza los usos del concepto de recuperación señalan que al considerar estas cinco dimensiones se puede comprender que algunos autores asimilen el concepto a la remisión (o aspecto clínico de la recuperación), otros a la funcionalidad, a cuestiones sociales o del cuidado físico, mientras que otros adoptan la perspectiva más propia de los usuarios, que Whitley y Drake (23) conceptualizan como “existencial”.

En consecuencia, la recuperación puede ser equiparada a una sola o a algunas de estas dimensiones, y en este sentido resulta problemática la posibilidad de analizar de manera tan fragmentaria una realidad que se caracteriza por su complejidad, con el riesgo de perder de vista que las dimensiones son establecidas a los fines analíticos y/o de evaluación del tratamiento pero no existen como realidades aisladas unas de otras. Con esto se quiere señalar que el sujeto no se encuentra social y funcionalmente enfermo y subjetivamente sano o a la inversa, entre otras posibles combinaciones; sino que todas ellas ocurren simultánea e inextricablemente en la misma persona al mismo tiempo, y son los profesionales quienes compartimentan esas dimensiones a los fines analíticos. Lo interesante del concepto -y las prácticas que éste supone- es que se coloca el acento en la capacidad de recuperarse que posee el propio sujeto con un padecimiento psíquico, no se trata de algo que pueda ser provisto por otro. Como lo señalan Agrest y Druetta (15) en esto se diferencia del concepto de "rehabilitación” en el campo de la Salud Mental, vinculado a lo que hacen los profesionales a favor de la recuperación de sus pacientes. En este sentido aparece en el relato del psiquiatra (espacio 2) la referencia a la rehabilitación de los pacientes como aquello que se realiza sobre quien tiene algún padecimiento. Por el contrario, la recuperación supone la participación activa del usuario de su proceso de cura. 
Pensar en la participación del “usuario con sufrimiento mental” en su recuperación, aún si ello supone dejar a un lado los diagnósticos (psicopatológicos y psiquiátricos), como ocurre en el espacio 1 (FA) contribuye a pensar los usuarios como sujetos de derecho, con un rol activo, capaz de producir un modelo de cuidado o más precisamente de autocuidado, orientado a su recuperación.

Al respecto, se puede señalar que la propuesta de incorporar a los pacientes en el gobierno de su salud ya había sido planteada por Tosquelles en Francia y Basaglia en Italia -aunque desde un posicionamiento vinculado a lo político-, ambos buscando constatar el efecto terapéutico de la participación de los pacientes en la gerencia de las Casas de Salud, Hospitales y otros servicios de salud mental. Ellos plantean que participar del gobierno de las instituciones influye en forma positiva sobre el proceso de cura de muchos enfermos, aumentando su capacidad de relación social e iniciativa. Para éstos el autogobierno no era solamente un principio político al cual adherían, representaba además un medio para la progresiva constitución de sujetos autónomos, capaces de sobrevivir a los conflictos y a las dificultades inherentes a la época y a la vida en sociedad (6). Subyace a esta perspectiva una concepción de sujeto capaz de gobernar su propia vida, administrar sus necesidades en su contexto social particular (aun cuando el hospital o casa de salud no sean espacios de pura autonomía).

Es en este sentido que Sousa Campos (7) va a señalar que curar a alguien será siempre luchar siempre para ampliar el coeficiente de autonomía de esa persona particular. Es así que será fundamental en cualquier proceso terapéutico el esfuerzo orientado para aumentar la capacidad de autonomía del paciente, para mejorar la comprensión de su propio cuerpo, de su enfermedad, de sus relaciones con el medio social y, en consecuencia, la capacidad de cada uno para instituir normas que le amplíen las posibilidades de sobrevivencia y la calidad de vida. El FA se vincula ideológicamente a tales desarrollos críticos del modelo asilar, que encuentran su auge en la década del 60’ y 70' con el desarrollo de la anti psiquiatría.

$\mathrm{Al}$ respecto resulta relevante lo planteado por Correa Urquiza (21) como resultado de un trabajo etnográfico sobre la experiencia de la Radio Nikosia (en Barcelona) de la que participan personas que han sido diagnosticadas con algún problema de salud mental. La propuesta de la radio parte de crear espacios donde se habilite la posibilidad de una suspensión semántica de las categorías diagnósticas que materializan el estigma social que sufren los pacientes psiquiátricos, cuyo discurso ha sido históricamente negado, para generar un espacio donde "el llamado loco pueda despojarse -al menos circunstancialmentede las consideraciones que giran en torno a la dimensión patológica y se enfrente a la opción de alcanzar nuevas derivaciones en el entramado de su identidad. Un terreno para habitar su diferencia” (21). En este sentido se plantea el concepto de des-enfermar que refiere "a la posibilidad de apartarse de una identidad de enfermo para acercarse más a una reflexión alrededor del dolor sin la necesidad de asumir una incapacidad” (21), no se trata de curar, sino de facilitar la construcción de una identidad fuera de la noción de dificultad, de incapacidad, que habilite otras posibilidades, una identidad asociada a la idea de autor que dé lugar a un nuevo rol.

\section{Consideraciones Finales}

A partir de lo planteado se puede señalar que no todas las propuestas de trabajo con el arte en el campo de la salud representan una verdadera alternativa a la lógica biomédica de atención al sufrimiento mental. Esto resulta especialmente cierto cuando se continúa tratando el objeto ontologizado de la medicina, la enfermedad psiquiátrica y pensando al enfermo como objeto pasivo de cuidados. En este sentido, coincidimos con Sousa Campos (7) cuando plantea que es como si la dolencia ocupara toda la personalidad, todo el cuerpo, todo el Ser del enfermo, se habla hasta de un arte psicopatológico para referirse a pinturas 
elaboradas por "enfermos mentales", como si la enfermedad sustituyera a la mente, o al cerebro, la voluntad, todo el Ser del sujeto con sufrimiento psíquico. Haciendo una analogía con el concepto de Institución Total de Goffman se podría hablar de Enfermedad Total.

Cuando la enfermedad es colocada entre paréntesis, el poder y etiquetamientos biomédicos quedan en suspenso, buscando evitar la iatrogenia de intervenciones exageradas. De esta forma se destaca la integridad como ser humano del sujeto, que puede actuar en relación a su propia salud, con capacidad de producir y no solo reproducir la estructura social y de significados. Es en el contexto del FA que se observa un posicionamiento diferente del sujeto ante la sociedad, como artista, lo que constituye la posibilidad de borrar la identidad históricamente producida de «enfermos mentales» para dar lugar a nuevas formas de pensarse y ser vistos.

El arte se convierte en instrumento de lucha y resistencia orientada a la transformación de las prácticas de atención en salud mental, al tiempo que a promover la creación de lazos sociales para los usuarios dentro y fuera de las instituciones, favoreciendo la producción de transformaciones individuales, colectivas y sociales.

Es en este sentido que planteamos la necesidad de des-etiquetar o des-enfermar, para dar lugar a la construcción de un nuevo rol, que se aparte de la identidad de enfermo, para dejar de tratar "Enfermos Totales". La propuesta se orienta a una transformación de las prácticas de cuidado y, en consecuencia, de las estructuras ideológicas que contribuyen al mantenimiento de la institucionalización y cronificación de los pacientes.

Esto exigirá de cada equipo de salud un abordaje más integral de cada caso, que supere prácticas biologicistas y reduccionistas que vienen degradando a la clínica. En este sentido, promover la recuperación se orientaría a que los individuos tengan relaciones de mayor la autonomía y más maduras con la enfermedad, con la prevención de la salud, con profesionales, con dispositivos tecnológicos y aun también con las instituciones de salud.

Amarante sintetiza la contribución de Basaglia diciendo que si la enfermedad se coloca entre paréntesis la mirada deja de ser exclusivamente técnica, exclusivamente clínica, entonces es la persona el objetivo de trabajo, y no la enfermedad. De esta manera el mayor énfasis no se pone en el "proceso de cura" sino en el proceso de invención de la salud y de la "reproducción social del paciente" (24). Souza Campos (7) señala que la perspectiva es bien fenomenológica, se sale del objeto ontologizado de la medicina - la enfermedady entra en su lugar el enfermo. Pero no es un enfermo en general, sino que es un sujeto concreto, social y subjetivamente constituido. Todo el énfasis está puesto en su existencia concreta y sobre la posibilidad de "inventarse la salud” para estos sujetos. Inventar salud implicará más que una intervención técnica y estará ligada a la reproducción social del paciente, noción que está profundamente influenciada por la perspectiva de ciudadanía activa y de protagonismo, que parte del reconocimiento del paciente como una persona con derechos, que al mismo tiempo debe hacer valer sus derechos (6).

Tales argumentos permiten plantear que, el otorgar un espacio diferente a quienes sufren un padecimiento psíquico, donde el diagnóstico no tiene lugar en relación a la actividad que se propone, donde es la propia iniciativa la que adquiere protagonismo, conduce a una elaboración subjetiva donde aparece la posibilidad de actuar sobre la propia realidad, desafiando vulnerabilidades y afirmando espacios propios.

\section{Agradecimientos}

A cada una de las personas entrevistadas, por su disposición a transmitir su experiencia.

A Eduardo Menéndez, por su acompañamiento en el trabajo de investigación, como director del proyecto.

Al CONICET, por el financiamiento recibido para desarrollar el proyecto. 


\section{Bibliografía}

1. Ferigato, S. Sy, A. y Resende Caravallho, S. 2011. Explorando las fronteras entre la clínica y el arte: relato de una experiencia junto al Frente de Artistas del Borda. Revista Salud Colectiva; 7(3): 347-363.

2. Hammersley, M. y Atkinson, P. 1994. Etnografía. Métodos de investigación. Barcelona: Ed. Paidos Ibérica.

3. Sy, A. 2013. La observación participante como técnica privilegiada en el acceso a la ritualización de prácticas en torno a la salud. A propósito de la propuesta del Frente de Artistas del Borda. Revista Papeles de Trabajo. Rosario: Centro de Estudios Interdisciplinarios en Etnolingüística y Antropología Socio-Cultural; 26:143-164.

4. Gándara, M.J. 2008. Psico-Neuro-Biología de la creatividad artística. Cuad. Psiquiatría. Comunitaria; 8(1): 29-46.

5. Guimón, J. 2008. Terapia por el Arte. Cuad. Psiquiatría Comunitaria; 8(1): 9-25.

6. Basaglia, F. 1999. La institución negada. Buenos Aires: Ed. Barral.

7. Sousa Campos, GW. 2001. Gestión en Salud. En defensa de la vida. Buenos Aires: Lugar Editorial.

8. Bolós AM, Hernández AMB, González AM. 2015. Arte como herramienta social y educativa. Revista Complutense de Educación; 26(2): 315-329.

9. Mosquera MI. 2015. Arte y comunicación, una alternativa de intervención para la enfermedad mental. Ciencia \& Salud; 3(12):29-36.

10. Abril M, Fernández CM. 2012. Impacto de la violencia intrafamiliar y de género en el desarrollo vital de la mujer con enfermedad Mental. Arte terapia; 7:97-114.

11. Muñoz Montejano, M., Crespo Sierra M.T. 2014. Arte terapia y migración: inclusión social a través de la apropiación simbólica del paisaje. Arte terapia; 9:13-24.

12. Marín Alaniz, J. 2014. Ayni: Por una infancia sin fronteras. Arte terapia con hijos de migrantes en el norte de Chile. Arte terapia; 9: 61-72.

13. Vanegas M., González M. 2014. Efecto de la actividad gráfica en las emociones y el estrés. Arte terapia; 9:145-154.

14. Domínguez Toscano, P.Má , López Martínez, Ma D. 2014. Efecto de la arte terapia en la ansiedad y depresión, la capacitación sociocultural y la reducción de la reincidencia penitenciaria de personas reclusas. Arte terapia; 9:39-60.

15. Agrest, M., \& Druetta, I. 2011. El concepto de recuperación: la importancia de la perspectiva y la participación de los usuarios. Vertex Revista Argentina de Psiquiatría; 22:56-64.

16. Castro, R. 2011. Teoría Social y Salud. Buenos Aires: Lugar Editorial.

17. Goffman, E. 1984. Internados: Ensayos sobre la situación social de los enfermos mentales. Buenos Aires: Ed. Amorrortu.

18. Becker, H. 1963. Outsiders: Studies in Sociology of Deviance. New York: Free Press of Glencoe.

19. Scheff, TJ. 1966. Being Mentally Ill: A Sociological Theory. Chicago: Aldine.

20. Ferreira, L. Martínez Hernáez, A. 2003. Ulisses, Greta y otras vidas: Tiempo, espacio y confinamiento en un hospital psiquiátrico del sur de Brasil. Cuadernos de Psiquiatría comunitaria; 3(1): 59-70.

21. Correa Urquiza, M. 2010. Radio Nikosia: la rebelión de los saberes profanos (otras prácticas, otros territorios para la locura). Tesis Doctoral. Universitat Rovira i Virgili.

22. Slade, M. 2009. 100 ways to support recovery. Disponible en: http://www.nevil. org.au/uploads/images/118/100_Ways_to_Support_Recovery.pdf (último acceso 27/04/2015)

23. Whitley R, Drake R. 2010. Recovery: A dimensional approach. Psychiatric Services; 61: 1248-1250.

24. Amarante, P. 1996. O homem e a serpente. Rio de Janeiro: Editora Fiocruz. 